\title{
Dynamics Analysis and Fractional-Order Approximate Entropy of Nonlinear Inventory Management Systems
}

\author{
Tengfei Lei $\left(\mathbb{D},{ }^{1}\right.$ Rita Yi Man $\mathrm{Li}^{2}$ and Haiyan Fu ${ }^{3}{ }^{3}$ \\ ${ }^{1}$ Rattanakosin International College of Creative Entrepreneurship, Rajamangala University of Technology Rattanakosin, \\ Rattanakosin, Bangkok, Thailand \\ ${ }^{2}$ Department of Economics and Finance, Hong Kong Shue Yan University, Hong Kong, China \\ ${ }^{3}$ Collaborative Innovation Center of Memristive Computing Application, Qilu Institute of Technology, Jinan 250200, \\ Shandong, China
}

Correspondence should be addressed to Haiyan Fu; fuhy413@126.com

Received 18 February 2021; Revised 17 March 2021; Accepted 29 March 2021; Published 8 April 2021

Academic Editor: Yi Qi

Copyright (c) 2021 Tengfei Lei et al. This is an open access article distributed under the Creative Commons Attribution License, which permits unrestricted use, distribution, and reproduction in any medium, provided the original work is properly cited.

Inventory management is complex nonlinear systems that are affected by various external factors, including course human action and policy. We study the inventory management model under special circumstances and analyse the equilibrium point of the system. The dynamics of the system is analysed by means of the eigenvalue trajectory, bifurcations, chaotic attractor, and largest Lyapunov exponent diagram. At the same time, according to the definition of fractional calculus, the fractional approximate entropy is used to analyse the system, and the results are consistent with those of the largest Lyapunov exponent diagram, which shows the effectiveness of this method.

\section{Introduction}

Over the past few decades, chaos and its applications have attracted remarkable consideration in a variety of fields, including chemistry, ecology, and economy [1]. An economics management system is a typical discrete chaotic system, which has widely concerned scientists [2-8]. In 1993, Stiles and Levy proposed the concept of chaotic strategic management in [3]. In 1994, Feichtinger et al. [4] studied many chaotic systems in the field of management operations research, such as queuing system, inventory system, and planning and scheduling system. These systems are mainly characterised by the chaos of queue, inventory, and planning and scheduling under different management decision rules. Murphy [5] used the chaos theory as a model to study the management of public relations, such as problems and crises. After summarising the research status of chaos management, Joseph [6] pointed out that chaos management depends on change rules, which are a set of rules based on orderly or disorderly changes, adaptability, and new orderly emergence process.
The fractional calculus theory has a history of more than 300 years. Its history is almost the same as that of traditional integral calculus theory. Compared with an integer-order differential equation, a fractional-order differential equation has a memory effect [9-14]; therefore, it can describe these natural phenomena more accurately [9]. Many fractionalorder chaotic systems, such as the fractional-order Lorenz system $[15,16]$ and the fractional-order Chen system $[17,18]$, have been studied [19]. However, most of the studies on chaos based on the theory of fractional calculus have been mainly limited to continuous-time chaotic systems, and the study of fractional-order discrete-time chaotic systems is insufficient. Thus, researchers turned their attention to apply the theory of discrete fractional calculus to discrete chaotic maps $[20,21]$. It has been proven that there are abundant dynamical behaviours in the fractional chaotic maps [22-27]. All of the above studies are based on chaotic systems, and other fields of fractional order have not been studied.

Many scientists have conducted considerable research on the complexity of time series such as economic 
management [8, 23-25]. Grassberger and Procaccia [28] used the GP algorithm to analyse the complexity of Hénon mapping, which was an early report on the complexity analysis of chaotic systems. Balasubramanian et al. [29] used the Lempel-Ziv algorithm and the ApEn algorithm to analyse the complexity of logistic mapping and used a complexity measure to distinguish among the different states of the system. In [30], the PE algorithm was used to analyse the complexity of chaotic pseudorandom sequences. In [31], the researchers analysed the randomness of discrete chaotic systems by using the SymEn algorithm and showed that discrete chaotic systems can be used as random sources. All of the above systems are based on the theory of integer-order differential information. Can we further extend them to the theory of fractional calculus?

In this paper, we propose a new special nonlinear inventory management system $[32,33]$. The discretisation of the proposed system was performed using a Matlab simulation. The rest of this paper is organised as follows. In Section 2, the equilibrium point and the $0-1$ test of the $2 \mathrm{D}$ inventory management nonlinear systems are presented. In Section 3, the bifurcation diagram, phase diagrams, and Lyapunov exponent spectrum are used to analyse the dynamics of the system. In Section 4, the fractional-order approximate entropy of 2D nonlinear inventory management systems is analysed. Finally, the obtained results are summarised in Section 5.

\section{Nonlinear Inventory Management Model Description}

2.1. 2D Inventory Management Model. Inventory management is an important part of enterprise organisation and management activities. A class of nonlinear inventory management systems is as follows [2]:

$$
\left\{\begin{array}{l}
x_{i+1}=s+p z_{i+1}, \\
y_{i+1}=q x_{i+1}+r y_{i} z_{i}, \\
z_{i+1}=1-x_{i}-y_{i}+z_{i},
\end{array}\right.
$$

where $s, p, q$, and $r$ are the system parameters, $s$ represents the initial sales base, $p$ represents the inventory fund transfer rate, $q$ represents the product resource rate, $r$ represents the inventory efficiency, $x_{i}$ represents the resources for sales in the time period $i, y_{i}$ represents the number of customers in the time period $i$, and $z_{i}$ represents the inventory capital of the company in the time period $i$. After normalising the parameters of the inventory management model, we obtained the following: $0<x_{i}<1,0<y_{i}<1$, and $0<z_{i}<1 / r$. Here, $p=0.43, q=0.38, s=0.11$, and $r=0.72$. The attractors of system (1) are shown in Figure 1.

The company had surplus inventory in stage $i$, and its main goal was to deal with this surplus inventory. At this time, the company shifted resources from production to sales. The new customers absorbed by the company in stage $i+1$ mainly depended on the direct investment in sales in stage $i$. In addition, some old customers had to be added to the total customer volume. If the company's inventory was insufficient and could not meet the requirements of the regular customers for timely pickup, some regular customers would disappear from the company's customer list. $x_{i}<0$ indicates that the company had no resources available for sale. The absolute value of $x_{i}$ implies that it had to borrow funds and promote production to supplement the sales resources, $y_{i}<0$ denoted losing customers, and $z_{i}<0$ denoted that the sales were very strong and the products were in short supply.

The problems of convenient calculation and original speed, in this paper, considers a special case of inventory management system, $P=0$, that is, there is no transfer of resources. The mathematical model of inventory management (1) is rewritten as

$$
\left\{\begin{array}{l}
y_{i+1}=q s+r y_{i} z_{i}, \\
z_{i+1}=1-s-y_{i}+z_{i},
\end{array}\right.
$$

where $0<x_{i}<1,0<y_{i}<1$, and $0<z_{i}<1 / r$. The definition of the parameters is the same as in formula (1). Here, $q=0.051$, $s=0.58$, and $r=0.72$. The attractors of system (2) are shown in Figure 2.

2.2. Equilibrium Stability Analysis. The fixed point of system (2) can be obtained by solving the following equations:

$$
\left\{\begin{array}{l}
y_{i}-q s+r y_{i} z_{i}=0, \\
z_{i}-1-s-y_{i}+z_{i}=0 .
\end{array}\right.
$$

Apparently, the fixed point can be obtained by setting $A=\left\{\left(y_{0}, z_{0}\right) \mid y_{0}=1-s, z_{0}=(1-s(1+q) / r(1-s))\right\}$. The Jacobian matrix $J$ at the equilibrium set can be expressed as follows:

$$
\mathbf{J}=\left[\begin{array}{cc}
\frac{1-s(1+q)}{1-s} & r-r s \\
-1 & 1
\end{array}\right]
$$

The systems' characteristic equation corresponding to matrix $J$ can be written as follows:

$$
\left(\lambda-1+\frac{q s}{1-s}\right)(\lambda-1)+r-r s=0 .
$$

From equation (7), we inferred that when $r<q s /$ $(1-s)^{2}$, at the fixed point $p_{0}$, system (2) is unstable. When $r>q s /(1-s)^{2}$, at the fixed point $p_{0}$, system (2) is stable. When $r=q s /(1-s)^{2}$, system (2) is at the bifurcation point.

2.3. $0-1$ Test. We implemented the $0-1$ test method. The $0-1$ test is a novel test approach to determine whether a given deterministic nonlinear dynamic system is chaotic. It was proposed by Sun et al. [31] and has already been successfully tested for integer-order chaotic systems [31] and fractional order chaotic systems with delay time [8]. If a set of discrete data $\varphi(n)(n=1,2,3, \ldots)$ represents a one-dimensional observable dataset obtained from the iterative, then the following two real-valued functions can be defined: 


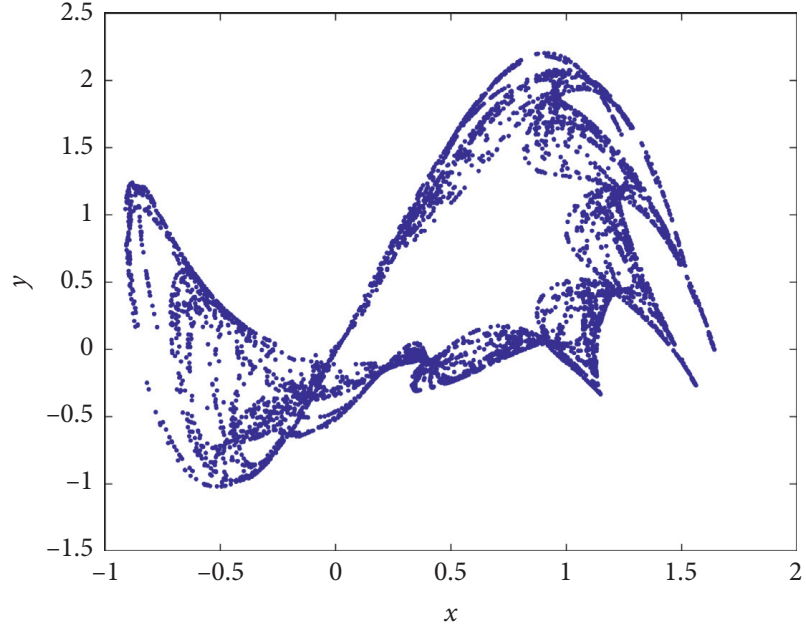

(a)

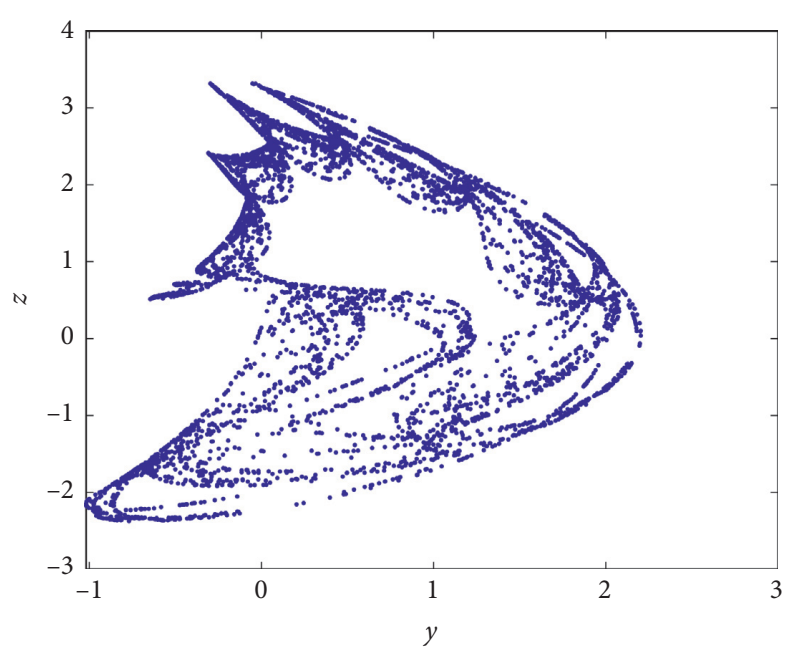

(b)

Figure 1: Attractors of system (1) with $p=0.43, q=0.38, s=0.11$, and $r=0.72$. (a) $x-y$ phase and (b) $y$ - $z$ phase.

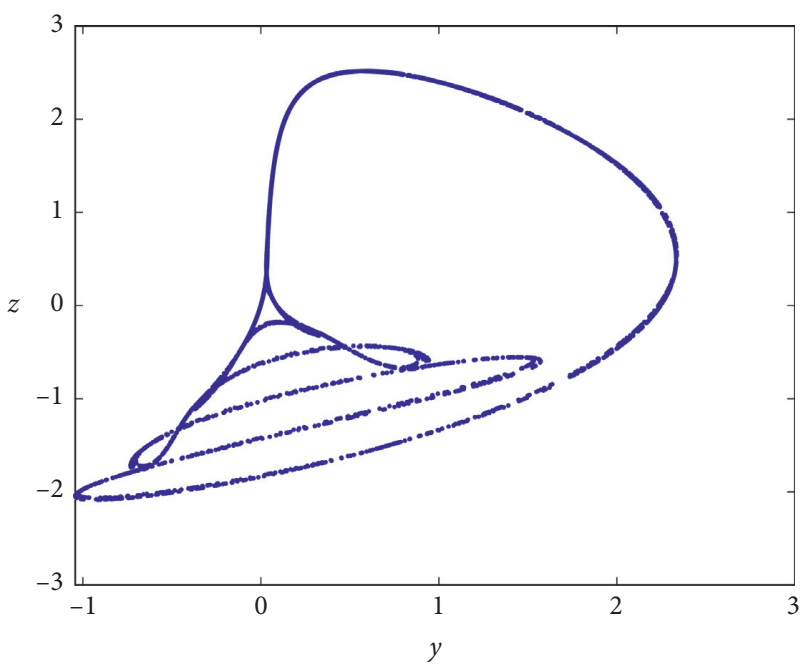

Figure 2: Attractors of system (2) from initial values (1.1, 0.1).

$$
\left\{p(n)=\sum_{i=1}^{n} \phi(i) \cos (\theta(i)) s(n)=\sum_{i=1}^{n} \phi(i) \sin (\theta(i))\right.
$$

where $\theta(i)=i \omega+\sum_{j=1}^{i} \phi(j)$, plotting the trajectories in the $p-s$ diagram. In any case, the trajectory in the $p$-s graph can provide a simple visual measurement. If the bounded trajectory in the $p$-s plots is a regular cloud slave, then the unbounded trajectory similar to Brownian motion indicates that the system is a chaotic system. This method was used to study the $y$ and $z$ sequence of the $2 \mathrm{D}$ system (2). Its parameters were the same as those shown in Figure 2. The obtained $p$-s diagram is shown in Figure 3; it can be seen that the system is chaotic.

\section{Analysis of Dynamic Characteristics}

3.1. Dynamics with $q$ Varying. We fixed $s=0.58$ and $r=0.72$ and let the derivative order $q$ vary from 0.05 to 0.08 with a step size of 0.0002 and the initial values of state variables $\left[y_{0}, z_{0}\right]=$
$[1.1,0.1]$. Figure 4 (a) shows that, upon the variation of $q$, it was obvious that the system chaos and periodic variation were repeated and crossed and were not a process from period to chaos directly. Obviously, we observed that $q \in[0.05$, $0.06) \cup[0.068,0.074] \cup[0.077,0.08]$ was in a chaos state and the other regions were in a chaotic state with periodic windows. Secondly, when $q \in[0.05,0.06) \cup[0.068,0.074] \cup[0.077$, 0.08], the largest Lyapunov exponent (LLE) was positive; LLE was equal to 0 in the other regions, as shown in Figure 4(b).

3.2. Dynamics with $r$ Varying. The chaotic system (2) had two system parameters besides $q$. In this study, the dynamic properties of the system parameters with $r$ varied were analysed. Firstly, the bifurcation diagrams of the 2D system with $r \in[0.5,0.75]$ were investigated, as shown in Figure 5(a). Obviously, we observed that $r \in[0.5,0.55) \cup[0.68,0.7]$ was in a chaos state and the other regions were in a chaotic state with periodic windows. Secondly, when $r \in[0.5$, $0.55) \cup[0.68,0.7]$, the LLE was positive; the LLE was equal to 0 in the other regions, as shown in Figure 5(b). Meanwhile, in eigenvalue trait of system (2) with $r$ varying, as shown in Figure $6, r$ ranges from 0.5 to 0.75 , and the eigenvalues are unstable, stable, and unstable processes, which are consistent with Figure 5. Eigenvalues are stable in the unit circle and unstable outside the unit circle.

For the case of $s=0.58, q=0.051$, and $r=0.6$, the periodic state phase diagram is given in Figure $7(\mathrm{a})$ and its corresponding $p$-s plot is presented in Figure 7(c). For the case of $s=0.58, q=0.051$, and $r=0.73$, the chaos state is shown in Figure 7(b) and the corresponding $p$-s plot is presented in Figure $7(\mathrm{~d})$. Thus, the system had different states with different parameters, and chaos was verified.

\section{Complexity Analysis of the System}

Entropy measures the complexity of a time series by measuring the probability of generating new patterns in signals [34-36]. The greater the probability of signal generation is, 


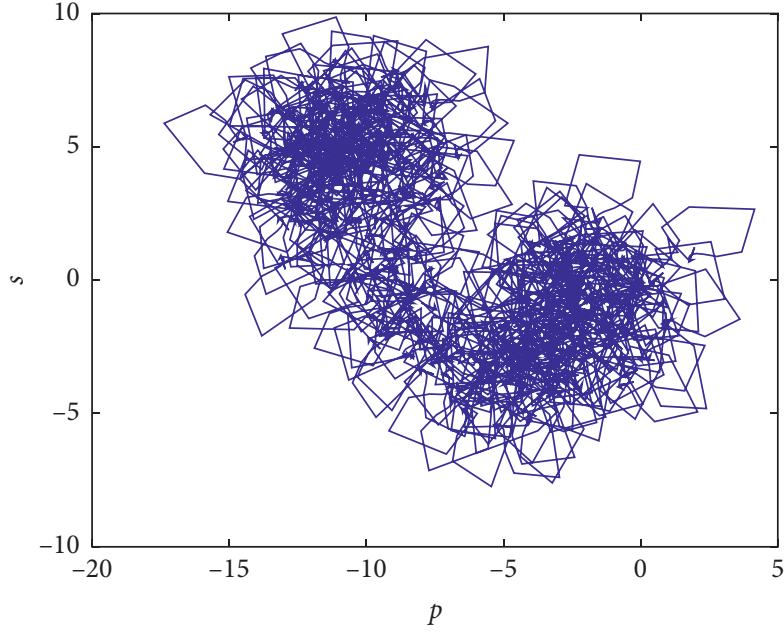

(a)

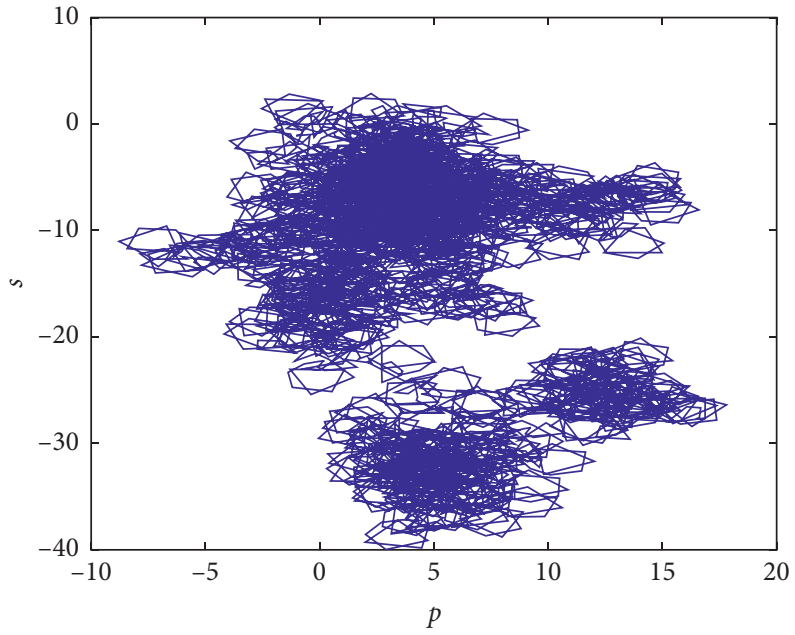

(b)

Figure 3: $p$-s phase diagrams of chaotic system (2) from initial values $(1.1,0.1)$. (a) $p$-s plot of $y$ sequence. (b) $p$-s plot of $z$ sequence.

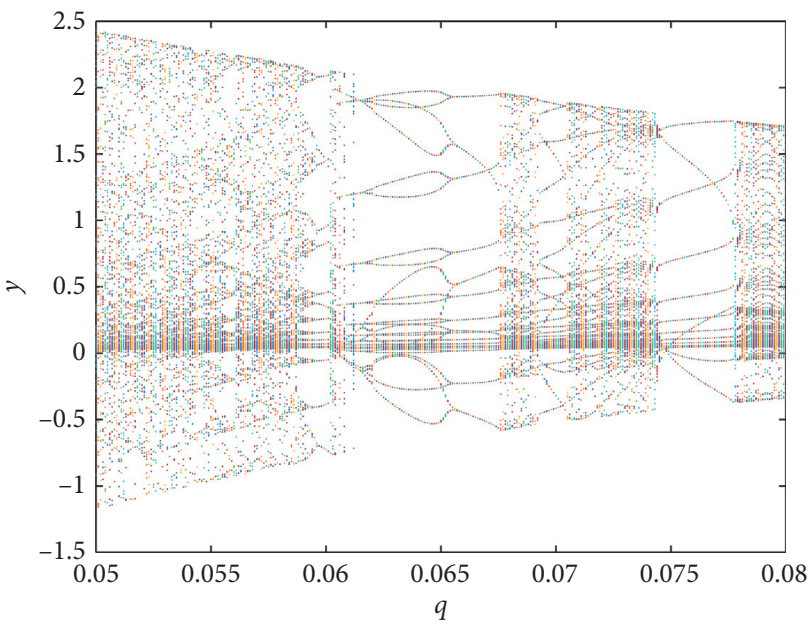

(a)

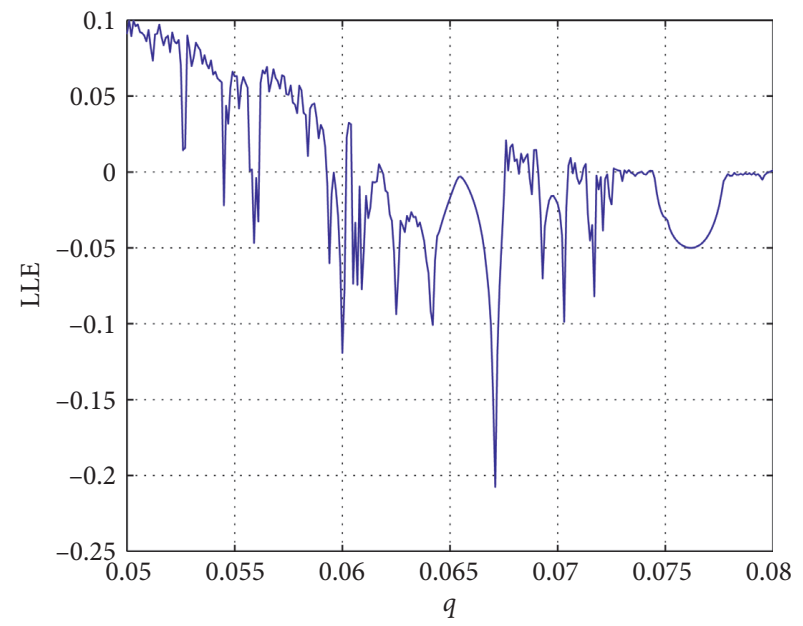

(b)

Figure 4: (a) Bifurcation diagram and (b) largest Lyapunov exponent (LLE) spectrum of system (2) with $q$ varying.

the greater is the value of the complexity of the sequence $[29,30]$.

4.1. Fractional Entropy Calculation. Information entropy can be calculated for both continuous and discrete signals and is defined as follows:

$$
H(p)=-c \sum_{i=1}^{n} P_{i} \ln P_{i} .
$$

Let $c=1$; then, according to the definition of a derivative,

$$
\begin{aligned}
\lim _{t \longrightarrow-1} \frac{d}{d t} \sum_{i=1}^{n} P_{i}^{-t} & =\lim _{t \longrightarrow-1} \sum_{i=1}^{n} \frac{d}{d t} P_{i}^{-t}=\lim _{t \longrightarrow-1} \sum_{i=1}^{n} P_{i}^{-t} \ln P_{i} \\
& =-\sum_{i=1}^{n} P_{i} \ln P_{i} .
\end{aligned}
$$

Comparing equation (7) with equation (8), we obtain the following:

$$
H(p)=\lim _{t \longrightarrow-1} \frac{d}{d t} \sum_{i=1}^{n} P_{i}^{-t} .
$$

According to [7], a fractional-order derivative can be defined as follows:

$$
\begin{aligned}
f^{\alpha}(x) & =D_{x}^{(\alpha)} f(x)=\lim _{h \rightarrow 0} L\left(\frac{f^{\alpha}(x+h)-f^{\alpha}(x)}{(x+h)^{\alpha}-x^{\alpha}}\right) \\
& =\lim _{h \rightarrow 0} \frac{\left(d\left(f^{\alpha}(x+h)-f^{\alpha}(x)\right) / d\left((x+h)^{\alpha}-x^{\alpha}\right)\right)}{\left(d\left((x+h)^{\alpha}-x^{\alpha}\right) / d h\right)} \\
& =\frac{f^{\prime}(x) f^{\alpha-1}(x)}{x^{\alpha-1}} .
\end{aligned}
$$




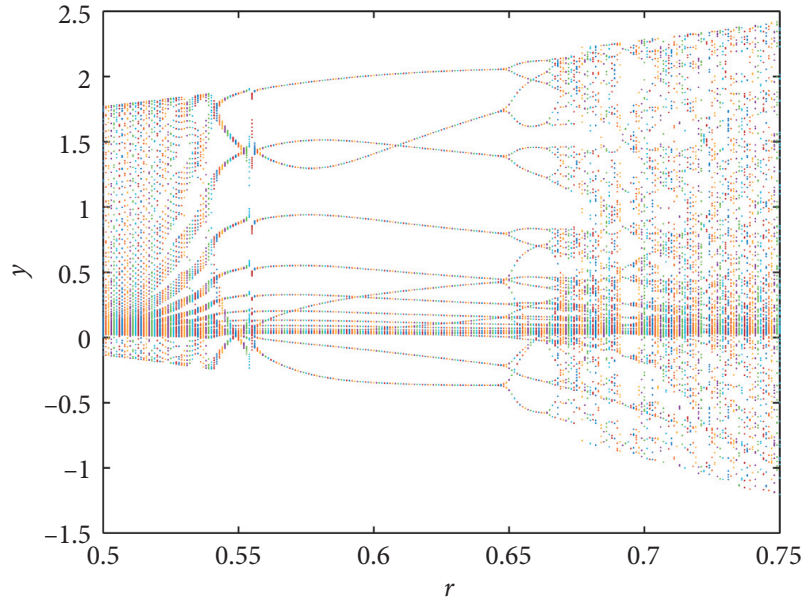

(a)

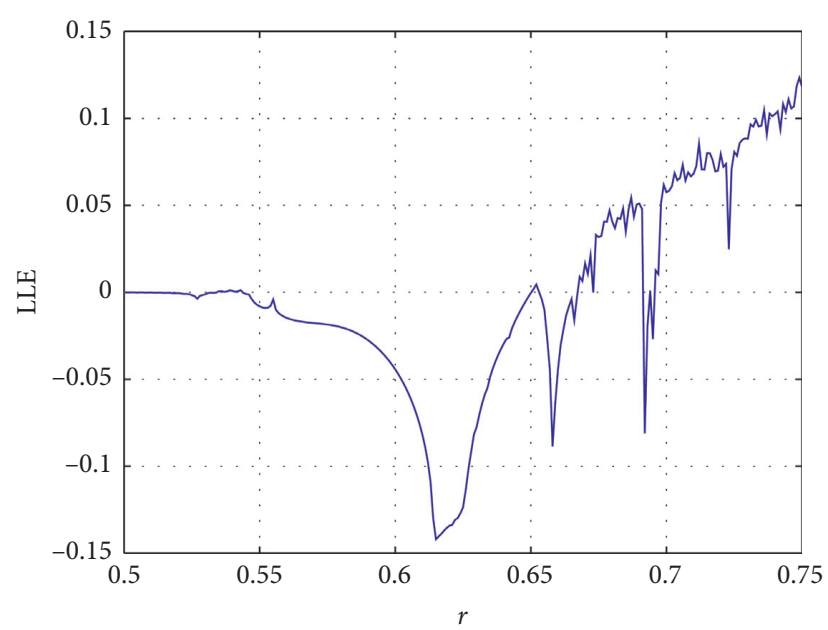

(b)

Figure 5: (a) Bifurcation diagram and (b) largest Lyapunov exponent (LLE) spectrum of system (2) with $r$ varying.

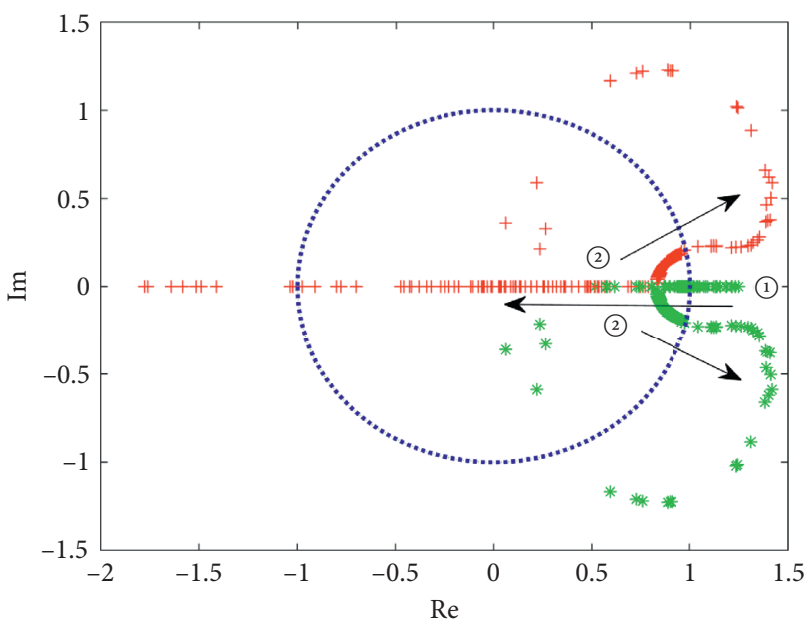

FIGURE 6: Eigenvalue trajectory of system (2) with $r$ varying.

Equation (10) was used to first derive $h$, and then, we set $h \longrightarrow 0$ to seek the limit. On the basis of this fractional derivative, KARCI proposed the first type of fractional derivative $[1,3]$ :

$$
H_{i}=D_{t}^{(\alpha)} P_{i}^{-t}=\sum_{i=1}^{n}\left|-p_{i}^{\alpha} p_{i} \ln p_{i}\right| .
$$

When $\alpha \longrightarrow 1$, equation (11) cannot be degenerated into equation (7), so there is an error. The fractional entropy is thus redefined as follows:

$$
\begin{aligned}
H^{\alpha}(P) & =\lim _{t \longrightarrow-1} \sum_{i=1}^{n} D_{x}^{(\alpha)} P_{i}^{-t}=\lim _{t \longrightarrow-1} \sum_{i=1}^{n} \frac{\left(-P_{i}^{-t} \ln \left(P_{i}\right) P_{i}^{-t}\right)^{\alpha-1}}{t^{\alpha-1}} \\
& =\lim _{t \longrightarrow-1} \sum_{i=1}^{n} \frac{-P_{i}^{-t \alpha} \ln \left(P_{i}\right)}{t^{\alpha-1}}=\lim _{t \longrightarrow-1} \sum_{i=1}^{n} \frac{-P_{i}^{\alpha} \ln \left(P_{i}\right)}{(-1)^{\alpha-1}} \\
& =(-1)^{\alpha} \sum_{i=1}^{n}-P_{i}^{\alpha} \ln \left(P_{i}\right) .
\end{aligned}
$$

When $\alpha \longrightarrow 1$, equation (12) can be degenerated into equation (7), but $0<\alpha \leq 1$ and $\mathrm{a}$ is a complex number. Thus, if $\quad(-1)^{-\alpha}=k_{1}+i k_{2}, \quad k_{1}, k_{2} \in R$; then, $H^{\alpha}(P)=$ $k_{1} \sum_{i=1}^{n} P_{i}^{\alpha} \ln \left(P_{i}\right)+\left[k_{2} \sum_{i=1}^{n} P_{i}^{\alpha} \ln \left(P_{i}\right)\right]$. If $k_{2}=0$, then $H^{\alpha}(p)$ is a real number. In practical applications, fractional entropy is redefined as follows:

$$
H^{\alpha}(p)=\operatorname{Max}\left\{k_{1} \sum_{i=1}^{n} P_{i}^{\alpha} \ln \left(P_{i}\right)\right\}, k_{2} \sum_{i=1}^{n} P_{i}^{\alpha} \ln \left(P_{i}\right) \text {. }
$$

4.2. Fractional-Order Approximate Entropy Calculation. Fractional-order entropy is combined with approximate entropy to obtain fractional-order approximate entropy (FAE). The specific steps are as follows.

In general, for the specific calculation method of the sample entropy of the time series $\{x(n)\}$ composed of $N$ data items, the specific steps are as follows:

S1: according to the serial number to form a one-dimensional $m$ vector sequence $\left\{X_{m}(1), \cdots, X_{m}(N-\right.$ $m+1)\}$, where $\quad X_{m}(1)=\{x(i), \quad x(i+1), \cdots$, $x(i+m-1)\}, 1 \leq i \leq N-m+1$. These vectors are $m$ consecutive $x$ values starting from point 1 .

S2: define the distance between $X_{m}(i)$ and $X_{m}(j)$, i.e., $d$ $\left[X_{m}(i) X_{m}(j)\right]$, as the difference between the maximum absolute values of the corresponding elements.

S3: define the criterion of similarity $r$. Calculate the value of $d\left[X_{m}(i), X_{m}(j)\right]<r(1 \leq j \leq N-m$, $1 \leq i \leq N-m)$ and its ratio to the total distance $N-m$, denoted as $B_{i}^{m}(r)=\left(B_{i} /(N-m-1)\right)$.

S4: on the basis of equation (12), the average value of $B_{i}^{m}(r)$ can be calculated as follows:

$$
B^{m}(r)=\frac{1}{N-m} \sum_{i=1}^{N-m}\left(-B_{i}^{m}(r)_{i}\right)^{\alpha} B_{i}^{m}(r) \ln B_{i}^{m}(r) .
$$




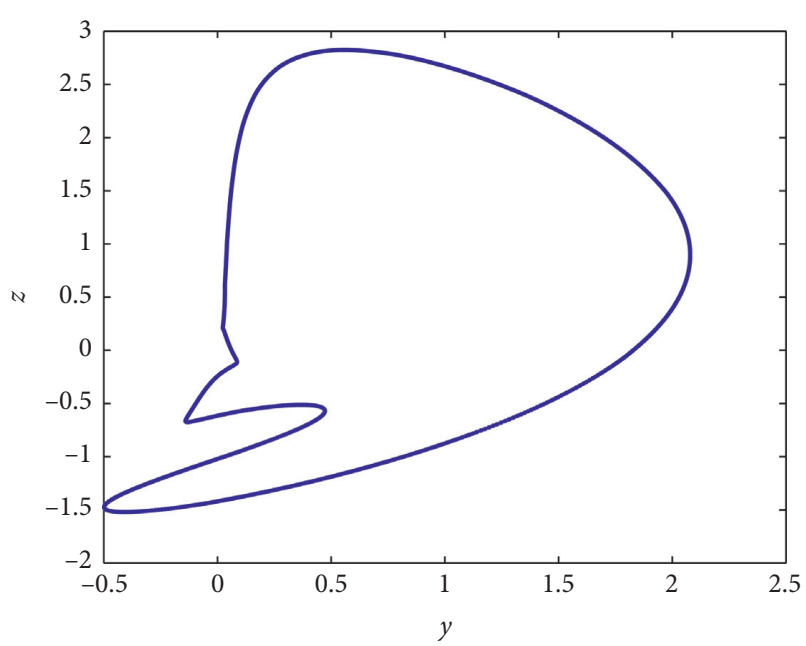

(a)

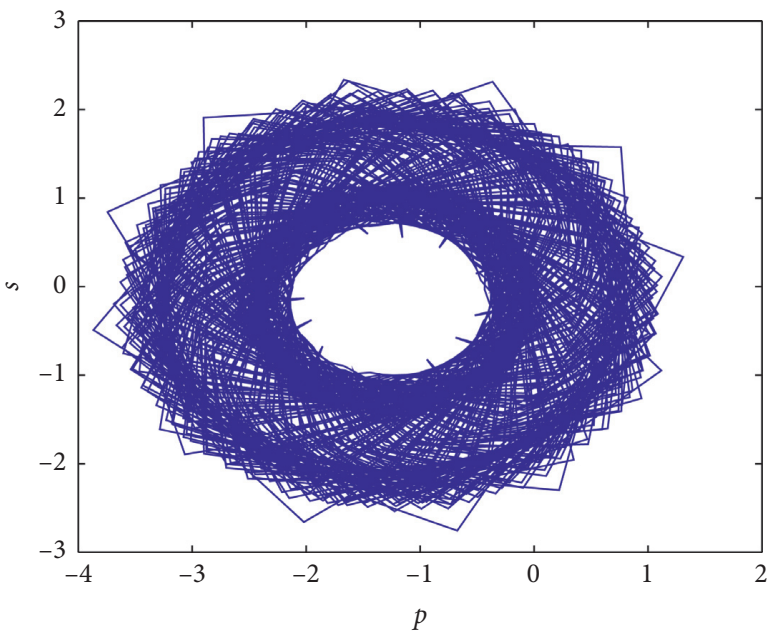

(c)

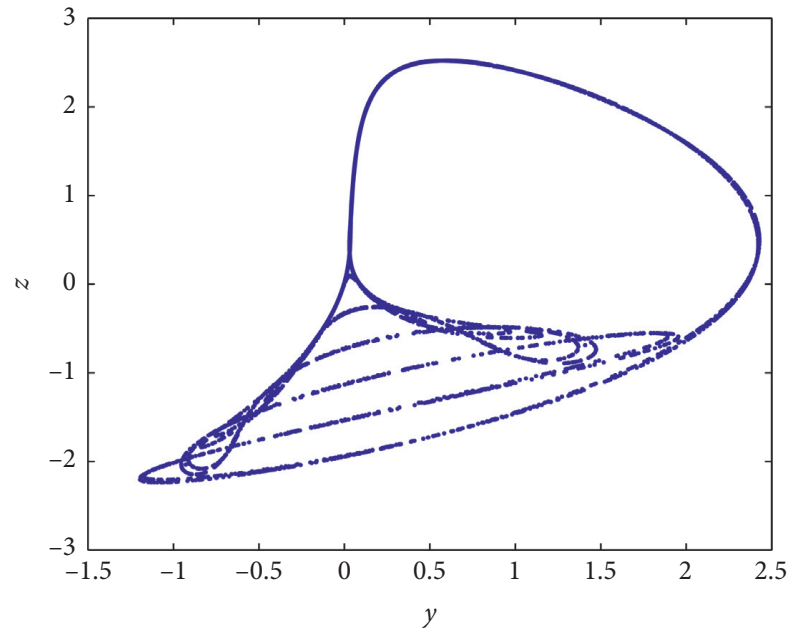

(b)

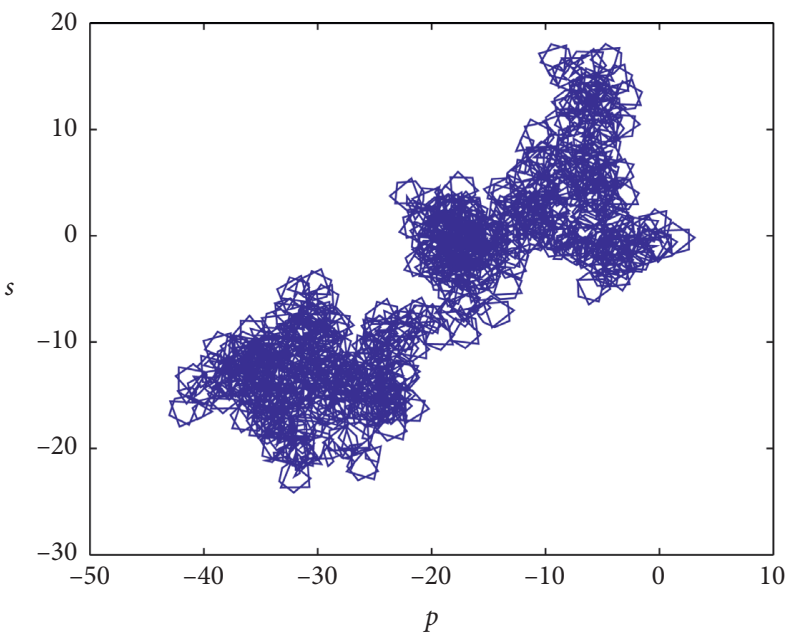

(d)

FIGURE 7: Phase diagrams and $p$-s plots of the systems with varying $r$ : (a) $y$ - $z$ phase with $r=0.6$, (b) $y$ - $z$ phase with $r=0.73$, (c) $p$-s plots with $r=0.6$, and (d) $p$-s plots with $r=0.73$.

S5: similarly, change $m$ to $m+1$, and repeat S1 to S3 to obtain $A^{m}(r)$.

S6: theoretically, according to equation (13), the FAE complexity can be calculated as follows:

$$
\begin{aligned}
\operatorname{FAE}(m, r)= & \max \left\{\operatorname{rel}\left(B^{m}(r)-\mathrm{A}^{m}(r)\right), \operatorname{imag}\left(B^{m}(r)\right.\right. \\
& \left.\left.-\mathrm{A}^{m}(r)\right)\right\} .
\end{aligned}
$$

4.3. Complexity Analysis. The FAE analysis results of the inventory management system with varying $q$ and $r$ values are shown in Figure 8. Here, the same parameter settings and step size are used as those in the corresponding bifurcation diagrams and LLE. As demonstrated in these
FAE plots, the results matched the corresponding bifurcation diagrams well. When the system was chaotic, higher complexity measurement results were obtained, whereas when the system was nonchaotic, the measured results were relatively small. Obviously, FAE provided different information on the dynamics of the system from that obtained from the bifurcation diagrams. Thus, we could determine the complexity variation trend of the system clearly.

As it is well known and quite obvious, we do not need chaos in the inventory management system, at least in most cases. This implies that the system is unstable and the inventory management situation has become unpredictable. We diagnosed the status of the inventory management system by means of the complexity method using the time series. 


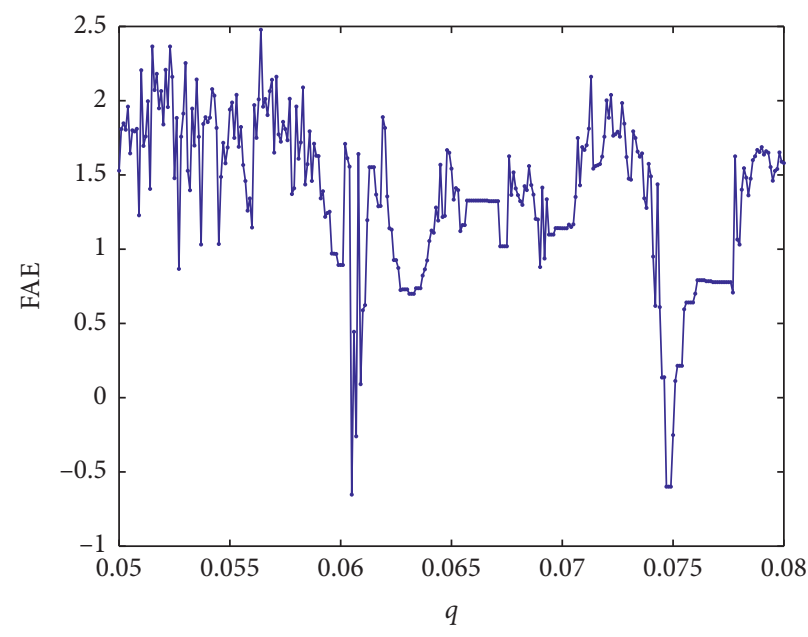

(a)

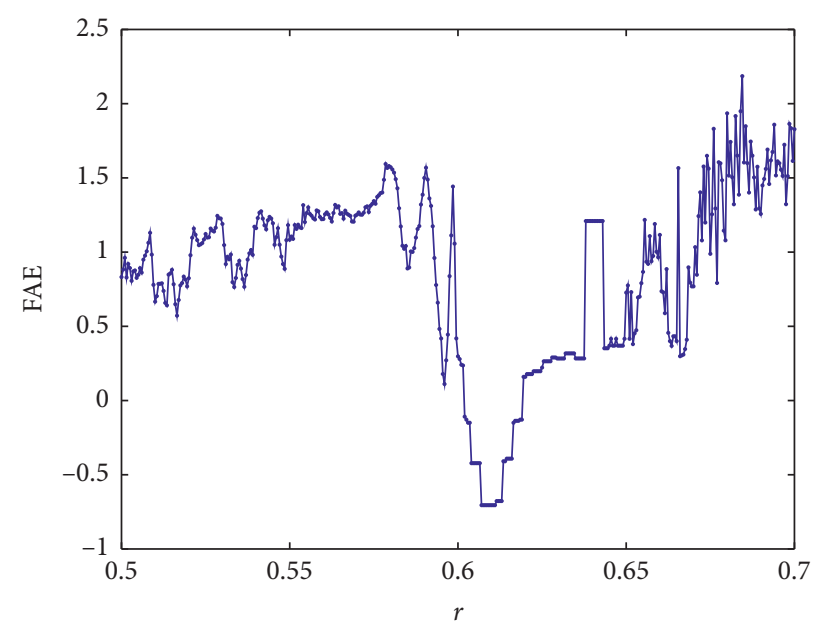

(b)

FIGURE 8: Fractional-order approximate entropy (FAE) of system (1) with (a) varying $q$ and (b) varying $r$ values.

\section{Conclusion}

In the present study, the dynamic behaviour of a nonlinear inventory management system was investigated. The different aspects of the dynamic behaviour of the system, including the phase diagrams and their corresponding $p-s$ plots, bifurcation diagrams, LLE with parameters and FAE complexity with parameters, were studied. We found that chaos and different states could be observed with different parameter settings. Meanwhile, the AEP complexity showed the changes in the complexity of the system. It also showed that the system had a wide region of high complexity in the corresponding parameter planes.

\section{Data Availability}

The data used to support the findings of this study are available from the corresponding author upon request.

\section{Conflicts of Interest}

The authors declare that they have no conflicts of interest.

\section{Acknowledgments}

This work was supported the Major Scientific and Technological Innovation Projects of Shandong Province (Grant no. 2019JZZY010111), Natural Science Foundation of Shandong Province (Grant no. ZR2017PA008), Key Research and Development Plan of Shandong Province (Grant no. 2019GGX104092), and Science and Technology Plan Projects of Universities of Shandong Province (Grant no. J18KA381).

\section{References}

[1] A. T. Azar and S. Vaidyanathan, Chaos Modeling and Control Systems Design, Springer International Publishing, Berlin, Germany, 2015.
[2] J. P. Pinder, "Nonlinear dynamical systems and inventory management," Managerial and Decision Economics, vol. 17, no. 1, pp. 27-43, 1996.

[3] D. Levy, "Chaos theory and strategy :theory, application, an managerial implications," Strategic Management Journal, vol. 15, pp. 167-178, 1994.

[4] G. Feichtinger, C. H. Hommes, and W. Herold, "Chaos in a simple deterministic queueing system," ZOR - Methods and Models of Operations Research, vol. 40, no. 1, pp. 109-119, 1994.

[5] P. Murphy, "Chaos theory as a model for managing issues and crises," Public Relations Review, vol. 22, no. 2, pp. 95-113, 1996.

[6] E. C. Joseph, "Chaos \& po stmodernism forecasting \& futuring insights," Technical report, Futurics, Summer-Fall, pp. 1-12, 1994.

[7] Y. Ding and J. Cao, "Bifurcation analysis and chaos switchover phenomenon in a nonlinear financial system with delay feedback," International Journal of Bifurcation \& Chaos, vol. 25, no. 12, pp. 2671-2691, 2015.

[8] S. Wang, S. He, A. Yousefpour, H. Jahanshahi, R. Repnik, and M. Perc, "Chaos and complexity in a fractional-order financial system with time delays," Chaos, Solitons \& Fractals, vol. 131, 2020.

[9] R. Hilfer, Applications of Fractional Calculus in Physics, World Scientific Press, New Jersey, NJ, USA, 2001.

[10] X. Yi, R. Guo, and Y. Qi, "Stabilization of chaotic systems with both uncertainty and disturbance by the UDE-based control method," IEEE Access, vol. 8, no. 1, pp. 62471-62477, 2020.

[11] L. Liu, B. Li, and R. Guo, "Consensus control for networked manipulators with switched parameters and topologies," IEEE Access, vol. 9, pp. 9209-9217, 2021.

[12] R. Peng, C. Jiang, and R. Guo, "Stabilization of a class of fractional order systems with both uncertainty and disturbance," IEEE Access, vol. 9, p. 42697, 2021.

[13] R. Xu and F. Zhang, " $\varepsilon$-Nash mean-field games for general linear-quadratic systems with applications," Automatica, vol. 114, pp. 1-6, 2020.

[14] T. Hou, Y. Liu, and F. Deng, "Finite horizon H2/Ho control for SDEs with infinite Markovian jumps," Nonlinear Analysis: Hybrid Systems, vol. 34, pp. 108-120, 2019. 
[15] Y. Yu, H.-X. Li, S. Wang, and J. Yu, "Dynamic analysis of a fractional-order Lorenz chaotic system «s," Chaos, Solitons \& Fractals, vol. 42, no. 2, pp. 1181-1189, 2009.

[16] S. B. He, K. H. Sun, and S. Banerjee, "Dynamical properties and complexity in fractional-order diffusionless Lorenz system," The European Physical Journal Plus volume, vol. 131, p. 254, 2016.

[17] J. G. Lu and G. Chen, "A note on the fractional-order Chen system," Chaos, Solitons \& Fractals, vol. 27, no. 3, pp. 685-688, 2006.

[18] A. S. Hegazi and A. E. Matouk, "Dynamical behaviors and synchronization in the fractional order hyperchaotic Chen system," Applied Mathematics Letters, vol. 24, no. 11, pp. 1938-1944, 2011.

[19] Y. X. Xu, K. H. Sun, S. B. He et al., "Dynamics of fractionalorder simplified unified system based on Adomian decomposition method," The European Physical Journal Plus volume, vol. 131, pp. 1-12, 2016.

[20] M. Edelman, "Fractional maps and fractional attractors part I: $\alpha$-families of maps," The interdisciplinary journal of Discontinuity Nonlinearity and Complexity, vol. 1, no. 4, pp. 305-324, 2012.

[21] M. Edelman, "Fractional maps and fractional attractors. Part II: fractional difference caputo $\alpha$ - families of maps," The Interdisciplinary Journal of Discontinuity, Nonlinearity, and Complexity, vol. 4, no. 4, pp. 391-402, 2015.

[22] G.-C. Wu and D. Baleanu, "Discrete fractional logistic map and its chaos," Nonlinear Dynamics, vol. 75, no. 1-2, pp. 283-287, 2014.

[23] G.-C. Wu, D. Baleanu, and S.-D. Zeng, "Discrete chaos in fractional sine and standard maps," Physics Letters A, vol. 378, no. 5-6, pp. 484-487, 2014.

[24] A.-A. Khennaoui, A. Ouannas, S. Bendoukha, X. Wang, and V.-T. Pham, "On chaos in the fractional-order discrete-time unified system and its control synchronization," Entropy, vol. 20, no. 7, p. 530, 2018.

[25] Y. Liu, "Chaotic synchronization between linearly coupled discrete fractional Hénon maps," Indian Journal of Physics, vol. 90, no. 3, pp. 313-317, 2016.

[26] M. K. Shukla and B. B. Sharma, "Investigation of chaos in fractional order generalized hyperchaotic Henon map," AEU International Journal of Electronics and Communications, vol. 78, pp. 265-273, 2017.

[27] Y. Ji, L. Lai, S. Zhong, and L. Zhang, "Bifurcation and chaos of a new discrete fractional-order logistic map," Communications in Nonlinear Science and Numerical Simulation, vol. 57, pp. 352-358, 2018.

[28] P. Grassberger and I. Procaccia, "Estimation of the Kolmogorov entropy from a chaotic signal," Physical Review A, vol. 28, no. 4, pp. 2591-2593, 1983.

[29] K. Balasubramanian, S. S. Nair, and N. Nagaraj, "Classification of periodic, chaotic and random sequences using approximate entropy and Lempel-Ziv complexity measures," Pramana, vol. 84, no. 3, pp. 365-372, 2015.

[30] E. H. Shen, Z. J. Cai, and F. J. Gu, "Mathematical foundation of a new complexity measure," Applied Mathematics \& $M e$ chanics, vol. 26, no. 9, pp. 1188-1196, 2005.

[31] K.-H. Sun, X. Liu, and C.-X. Zhu, "The 0-1 test algorithm for chaos and its applications," Chinese Physics B, vol. 19, no. 11, p. 110510, 2010.

[32] M. Sarkar and B. Sarkar, "Optimization of safety stock under controllable production rate and energy consumption in an automated smart production management," Energies, vol. 12, no. 11, p. 2059, 2019.
[33] J. Pinho, J. Resende, and I. Soares, "Capacity investment in electricity markets under supply and demand uncertainty," Energy, vol. 150, pp. 1006-1017, 2018.

[34] J. S. Richman and J. R. Moorman, "Physiological time-series analysis using approximate entropy and sample entropy," American Journal of Physiology Heart \& Circulatory Physiology, vol. 278, no. 6, pp. 2039-2049, 2000.

[35] W. Chen, J. Zhuang, W. Yu, and Z. Wang, "Measuring complexity using FuzzyEn, ApEn, and SampEn," Medical Engineering \& Physics, vol. 31, no. 1, pp. 61-68, 2009.

[36] S. Pincus, "Approximate entropy (ApEn) as a complexity measure," Chaos: An Interdisciplinary Journal of Nonlinear Science, vol. 5, no. 1, pp. 110-117, 1995. 